\title{
Little evidence for a selective advantage of armour-reduced threespined stickleback individuals in an invertebrate predation experiment
}

\author{
Michael Zeller • Kay Lucek • Marcel Haesler • \\ Ole Seehausen $\cdot$ Arjun Sivasundar
}

Received: 23 August 2011/ Accepted: 3 March 2012

(C) Springer Science+Business Media B.V. 2012

\begin{abstract}
The repeated colonization of freshwater habitats by the ancestrally marine threespined stickleback Gasterosteus aculeatus has been associated with many instances of parallel reduction in armour traits, most notably number of lateral plates. The change in predation regime from marine systems, dominated by gape-limited predators such as piscivorous fishes, to freshwater habitats where grappling invertebrate predators such as insect larvae can dominate the predation regime, has been hypothesized as a driving force. Here we experimentally test the hypothesis that stickleback with reduced armour possess a selective advantage in the face of predation by invertebrates, using a natural population of stickleback that is highly polymorphic for armour traits and a common invertebrate predator from the same location. Our results provide no compelling evidence for selection in this particular predator-prey interaction. We suggest that the postulated selective advantage of low armour in the face of invertebrate predation may not be universal.
\end{abstract}

Keywords $E d a \cdot \operatorname{Stn} 382 \cdot$ Aeshna $\cdot$ Dragonfly larvae $\cdot$ Lateral plates

\section{Introduction}

Testing the role of natural selection in phenotypic adaptations to divergent environments has proven to be a difficult task (Endler 1986; Conner 2001). Differences in resources, habitat structure, predation and parasite infestation and competition are often thought to be responsible for divergent natural selection. The latter, a key driver of adaptive phenotypic

Electronic supplementary material The online version of this article (doi:10.1007/s10682-012-9566-2) contains supplementary material, which is available to authorized users.

M. Zeller · K. Lucek · M. Haesler · O. Seehausen · A. Sivasundar ( $\square)$

Institute for Ecology and Evolution, University of Bern, Baltzerstrasse 6, 3012 Bern, Switzerland e-mail: arjun.sivasundar@iee.unibe.ch

M. Zeller · K. Lucek · M. Haesler · O. Seehausen · A. Sivasundar

Department of Fish Ecology, EAWAG Centre for Ecology, Evolution and Biogeochemistry,

Seestrasse 79, 6047 Kastanienbaum, Switzerland 
differentiation, can lead to ecological speciation (Rundle and Nosil 2005) and eventually adaptive radiation (Schluter 2000). Phenotypic differentiation driven by heterogeneity in habitat structure, available resources as well as intra- and inter-specific competition has been extensively studied and documented in a wide range of organisms (Schluter 2000). However, there is limited direct evidence for a role of predation leading to speciation (Vamosi 2005; Langerhans et al. 2007; Marchinko 2009). It has been shown that predators may cause divergent selection on body shape, for instance in the Bahamas mosquito fish Gambusia hubbsi, where divergent predation pressures have shifted morphological traits in different directions. Fish in low predation areas tend to have a more streamlined body whereas the fish in high predation regimes have a deeper body shape. These traits were found to be the basis for assortative mating, reproductive isolation and consequently speciation (Langerhans et al. 2007). Furthermore, it has been shown that predators can shape the evolution of divergent life histories (Reznick and Endler 1982; Johnson 2001). For example, increased predation rates on old or large individuals should favor the evolution of early maturation and a high reproductive effort, while contrary trends are expected by an increased rate of predation on very young age-classes and small individuals (Ernande et al. 2004; Gårdmark and Dieckmann 2006; Walsh and Reznick 2009).

Repeated parallel cases of invasion of freshwater habitats, and the presence of replicate sets of coexisting and divergent forms have made the threespined stickleback an important model system to study adaptive diversification and perhaps the early stages of speciation (McKinnon and Rundle 2002). They exhibit a range of phenotypic traits that are related to their defense against predators. These include external bony skeletal armour traits, consisting of lateral plates (which are modified scales), pelvic and dorsal spines and a pelvic girdle. Lateral plates can be divided into structural and non-structural plates; structural plates form, together with the pelvic girdle, pelvic spines and dorsal spines, a massive defensive structure. The presence of structural plates on both sides of the fish is crucial to the effectiveness of the spines against predators. Non-structural plates reduce the probability of integumentary injuries following an attack by a predator (Reimchen 1992a, b). In their ancestral marine habitat, stickleback are generally heavily armoured with a full set of lateral plates covering the whole flank of the body and have long dorsal and pelvic spines (Reimchen 1994; McKinnon and Rundle 2002). This extensive armour is thought to protect them in the marine habitat, which is dominated by gape limited and puncturing fish and bird predators (Gross 1978). Since the last retreat of the glaciers less than 15,000 years ago, the threespined stickleback has invaded temperate freshwater systems independently throughout the northern hemisphere (Bell and Foster 1994; McKinnon and Rundle 2002). Armour reduction has occurred repeatedly in most of these locations within this short evolutionary time. Various hypotheses have been postulated to explain the reduction in armour in freshwater relating to both biotic and abiotic factors (Barrett 2010). Biotic mechanisms include adaptations to different predator regimes (e.g. Reimchen 1980; Reimchen 1992a, b; Vamosi 2002; Marchinko 2009), food availability (Bjærke et al. 2010) and buoyancy (Myhre and Klepaker 2009), while abiotic mechanisms include adaptations to water chemistry (e.g. Heuts 1947; Giles 1983; Bell et al. 1993; Marchinko and Schluter 2007). However, in most cases, more than one factor is likely involved, and the reason for the observed pattern is difficult to single out.

The selective advantage of putative defense traits has been investigated and tested in several studies. Reimchen (1992a, b, 1994) found a positive correlation between the number of predatory fish species in a habitat and the length of pelvic and dorsal spines of sticklebacks. Most predatory fish are gape-limited, meaning that they are only able to eat prey items smaller than their own gape. Longer spines increase the effective diameter of an 
individual, making it more difficult for gape-limited fish and bird predators to swallow the prey (Bańbura 1994) and also increase the likelihood of injuries to the predator. Lateral plates increase the survival of sticklebacks following escape from attacks by a fish or bird predator (Hoogland et al. 1956; Reimchen 1992a, b; Reimchen 2000). In general, armour traits are thought to be important in marine and lacustrine habitats, where the predation regime is dominated by gape limited predators. However the mechanisms underlying the occurrence of populations fixed for the low plated phenotype in freshwater bodies, and the reason for its repeated parallel evolution remains poorly understood. Reimchen (1980, 1994) hypothesized that armour traits provide structures where grappling predators, such as dragonfly larvae, backswimmers (Notonecta sp.) and diving beetles, could grip their prey. Specifically, Reimchen (1980) suggested that spines might serve as objects that invertebrate predators such as Aeshna could use to grasp the fish; therefore a reduction in the spine phenotype (both in size and number) might confer a selective advantage in the face of strong dragonfly predation. He also suggested that external structures that enhance frictional contact with grappling predators, such as Aeshna, would be disadvantageous in such predation regimes. We tested the hypothesis that lateral plates represent such structures, and hence expect that low plating should be advantageous in an Aeshna dominated predation regime. Then, in shallow and stagnant water habitats where fish predators are uncommon and aquatic insects are important predators, low plate number, smaller plate size and reduced spines should be advantageous. An experimental test of this hypothesis (Marchinko 2009) showed that insect predation resulted in selection for faster growth rate, shorter spines, reduced girdle length, and selection for the allele associated with the low plate phenotype, but could not demonstrate selection on the lateral plate phenotype itself. In fact, the experiment only used juvenile fish, where the plate phenotype was not yet fully expressed. He did, though, find a selective advantage for the L-allele and argued that Ectodysplasin (Eda) might have pleiotropic effects that are under selection.

The genetic basis of the plate phenotype in stickleback is relatively well understood. A single gene, $E d a$, has been demonstrated to explain $\sim 75 \%$ of the genetic variation for different bony lateral plate phenotypes between marine and freshwater threespined stickleback (Colosimo et al. 2004). Changes within and near Eda have been shown to be responsible for the repeated loss of lateral plates in freshwater all over the world (Cresko et al. 2004; Colosimo et al. 2005). Phylogenetic analyses of the Eda sequences grouped most populations all over the world according to their plate phenotype. The same analyses with 25 neutral microsatellite markers grouped the populations by geography rather than plate morph suggesting that the Eda locus is a likely target of selection in freshwater habitats. There are two Eda alleles, referred to as the "L-"(low) and "C-" (complete) allele. Alleles for the low plated phenotype must have been maintained at low frequencies in marine stickleback (Schluter and Conte 2009). These freshwater alleles are exported to the sea through occasional hybridization events during contact between freshwater-resident and anadromous marine populations. As a consequence, freshwater alleles are now present as rare standing genetic variation in the sea, but can become positively selected for again during the colonization of new freshwater habitats (Cresko et al. 2004; Colosimo et al. 2005). However there is still only very limited empirical support for invertebrate predation induced selection on defense traits and their genetic components.

Despite decades of research, there remain significant gaps in our understanding of the potential of invertebrate predator induced selection on armour variants in adult threespined stickleback. The aim of the study is to test experimentally whether predation by dragonfly larvae exerts selection on armour related traits by comparing the distribution of armour variants and $E d a$ alleles between different experimental predation treatments. We exposed 
fish from a natural population that is highly polymorphic for defense-related traits to high densities of invertebrate predators (dragonfly larvae) and compared fish that survived and dead fish with those from a no predation control. The expectation was that low plated fish and/or those with reduced pelvic and dorsal spines have elevated survival rates under dragon fly predation.

We test these hypotheses in a highly polymorphic wild population. Threespined stickleback have spread rapidly across Switzerland in the last 140 years, following introductions from different European sources and hybridization, resulting in large phenotypic and genetic diversity within some populations (and also between populations; Lucek et al. 2010). This makes Swiss stickleback populations an ideal study system to address key questions in the processes involved in adaptive diversification during a biological invasion. Hybridization between different lineages during secondary contact in the Bernese midlands has been documented (Lucek et al. 2010) and the great diversity in defense related phenotypes makes these hybrid populations ideal to investigate these issues.

\section{Materials and methods}

Population based selection experiments

\section{Fish and dragonfly samples}

A total of 960 threespined stickleback were used for the experiment. All were taken from a natural population living in a stream near Bern, Switzerland. This population shows large variation in plate morphology and the length of pelvic and dorsal spines (Lucek et al. 2010). All fish were caught using either minnow traps or hand nets in spring 2010. The dragonfly larvae were collected using hand nets from the same site and other nearby ponds and streams. All dragonfly larvae used belonged to the genus Aeshna.

\section{Experimental setup}

Eight experimental ponds of approximately $3 \mathrm{~m} \times 2 \mathrm{~m}$ and $40 \mathrm{~cm}$ depth were each divided into two equal halves with nylon netting $(2 \mathrm{~mm}$ mesh size). Half-ponds were randomly assigned to the treatment (i.e. predation) or the control (no predation) group. Ten artificial plants, made of black plastic strips attached to a stone, and a wooden stick (about $0.5 \mathrm{~m}$ in length) were placed in each half-pond to provide shelter. The ponds were located within metal grill enclosures, so predation by bird or mammalian piscivores was not possible. Thirty adult stickleback were randomly assigned to each of the 16 half-ponds and allowed to acclimate to the environment for 2 days without predators. The fish were fed daily with a mixture of frozen Daphnia spp. and frozen chironomid larvae. After the acclimation period, ten dragonfly larvae (Aeshna spp.) were introduced to the experimental treatments. Ponds were checked twice a day and the remains of all dead fish were removed and preserved in $95 \%$ ethanol. Dead or ecclosed dragonfly larvae were replaced with new ones to keep the predator density constant. The experiment was conducted in two runs: the first from 4th to 15th of May 2010 and the second from 19th to 29th of May 2010. After each run, all ponds were emptied and surviving fish were euthanized with an overdose of clove oil and stored in $95 \%$ ethanol for further analysis. 


\section{Morphological analyses}

Standard length (SL), first dorsal spine length (FSL), second dorsal spine length (SSL), pelvic spine length (PSL) and body depth (BD, measured at the base of the second dorsal spine) were measured to the nearest $0.01 \mathrm{~mm}$ using digital calipers (Sylvac SA). All individuals were stained with alizarin red to ensure accurate counts of the lateral plates (Peichel et al. 2001), followed by removal of melanin pigments by bleaching in $3 \% \mathrm{H}_{2} \mathrm{O}_{2}$ for approximately $2 \mathrm{~h}$. Lateral plates were counted on the left side of each fish, and individuals were classified as low-, partially- or fully-plated phenotypes. Low plated individuals have between four and nine structural plates in the anterior region of the body, whereas fully plated individuals have continuous plating from the anterior region to the end of the caudal peduncle, forming a keel. Partial plated fish are intermediate, and either have a keel or lack one.

\section{Age determination}

A common way to make an accurate age determination for many fish species is with the use of scales (Helfman 1997); in stickleback, however, scales have evolved into lateral plates, which cannot be used for age determination. Otoliths, which are structures made of calcium carbonate in the inner ear, were used instead for age estimation. The accretion of the otoliths depends on the growth of the fish. Individuals generally show a higher growth rate in summer than in winter, resulting in seasonal rings on the otolith. By counting these rings, it is possible to determine the age of the fish in years. A procedure similar to the one described by Münzing (1959) was used to extract otoliths. Otoliths were extracted from ten randomly chosen fish from each control and each treatment, totalling 160 individuals from controls and 160 from treatments. Otoliths were mounted on slides and investigated twice under a microscope (Leitz Laborlux 11) by two different observers. Deviations between the different observers were discussed and investigated again to find an optimal determination of age.

\section{Genetic analyses}

All individuals were genotyped for two markers, Stn382 and Idh, using PCR protocols following Colosimo et al. (2005). The Stn382 microsatellite flanks a $60 \mathrm{bp}$ indel in intron 1 of the Eda gene, yielding either a 158 bp allele (low, or L, allele), associated with the low plated phenotype or a 218 bp allele (complete, or C, allele), associated with the complete plated phenotype. The $I d h$ microsatellite marker (Peichel et al. 2004) is diagnostic for sex, females being homozygous for a $300 \mathrm{bp}$ allele and males heterozygous for the $300 \mathrm{bp}$ allele and a $270 \mathrm{bp}$ allele. For DNA extraction, a small piece of pectoral fin tissue from each fish was placed into a tube with $180 \mu \mathrm{l}$ of $10 \%$ Chelex $100^{\circledR}$ (Biorad, USA). The tubes were incubated twice for $15 \mathrm{~min}$ at $95{ }^{\circ} \mathrm{C}$ each time, briefly vortexed between steps, spun down and $1 \mu \mathrm{l}$ of the supernatant was transferred into new tubes. PCR amplifications were performed in $10 \mu \mathrm{l}$ volumes. Details of PCR conditions are available upon request. The PCR products were run on a $1.5 \%$ agarose gel and genotypes scored by eye.

\section{Plate cover}

Not only does the number of lateral plates vary considerably among individuals in the studied population, so does the size of the plates. Thus, in addition to lateral plate counts, the area covered by lateral plates relative to the total surface area of the sides of the body 
was measured for a subset of samples. Thirty-nine randomly selected fish were scanned with a standard flatbed scanner. The surface area covered by plates as well as the total surface area of the fish was measured using Photoshop CS5 (Adobe, USA). Plate cover was then calculated as the ratio of the pixel counts of the two values.

\section{Statistical analyses}

Given that all linear measurements are correlated with body size, we first regressed measurements for each trait against SL and retained the residuals for all subsequent analyses. The distributions of all linear morphological traits (including SL) as well as plate counts were tested for normality using a one-sample Kolmogorov-Smirnov test for control and treatment independently. To account for potential differences among runs and ponds, a linear mixed model was used correcting for heterogeneity among runs. All traits were independently analyzed with treatment (predation and no predation controls) as explanatory variable and ponds nested within runs as random factors. This analysis was performed for lateral plate numbers and size corrected linear morphological traits, as well as the scores along the first two axes from principal component analysis (PCA) based on size corrected linear morphology (excluding plate count).

Standardized selection differentials $(i)$ for individual traits in each replicate were calculated according to the following equation (Endler 1986)

$$
i=\frac{\bar{X}_{a}-\bar{X}_{b}}{\sqrt{\operatorname{var}_{b}}},
$$

where $\bar{X}_{a}$ represents the mean trait value for fish under selection (treatment), $\bar{X}_{b}$ the mean trait value without selection (control), and $v a r_{b}$ is the trait variance in the control. Although the original equation compares the same group before and after selection, we use the control versus treatment approach rather than pre- and post-selection, assuming that the control represents the pre-selection distribution of traits. Since all experimental fish were randomly drawn from a much larger pool of thousands of fish, it is unlikely that there were large and systematic differences between the two groups at the start of the experiment. The reason we did not measure fish pre-selection was to avoid handling, and therefore stressing and possibly injuring fish, while taking measurements. The traits that we measured in this experiment require considerable handling, and while this would have been stressful or harmful to all fish, it would have been inordinately so for smaller individuals, thereby confounding all our analyses. To accurately count plates, especially on a small fish, requires the use of a dissecting needle, and the most accurate way is to stain the fish in alizarin (which we did), which is why we only did it at the end of the experiment. A similar approach was employed by Marchinko (2009) in a comparable experiment. Significance was estimated using a re-sampling procedure with 1,000 replicates. These analyses were performed using R 2.12.1 (The R Project).

\section{Individual based selection experiments}

\section{Experimental setup and procedure}

The experiments described above were performed with adult fish and used dragonfly larvae larger than $42 \mathrm{~mm}$. To examine the effect of predator size, prey size and the difference between them on predation success, we performed one-on-one trials using individuals 
taken from a broad size distribution of both predators and prey. We conducted 50 such trials using dragonfly larvae between 36 and $52 \mathrm{~mm}$ in length and fish between 25 and $44 \mathrm{~mm}$ SL. In each trial, one randomly chosen fish was exposed to one randomly chosen dragonfly larva for a maximum of $30 \mathrm{~min}$. Each trial was filmed and predator attack events were recorded. The dragonfly larvae were first placed in an aquarium $(130 \mathrm{~mm} \times 150 \mathrm{~mm} \times 125 \mathrm{~mm})$. After five minutes, the fish was added to the aquarium in an inverted transparent perforated plastic beaker in such a way that visual and chemical communication between predator and prey was possible, but actual predation was not. After another five minutes the beaker was removed. The trial was stopped five minutes later if the predator had successfully attacked a fish. If the dragonfly larva did not attempt to attack the fish within $30 \mathrm{~min}$, the trial was stopped. The outcome of each trial was assigned to one of three categories: 1-the dragonfly larva did not attack the prey; 2 - the dragonfly larva actively attacked the stickleback but had no success; 3 - the dragonfly larva successfully hunted the stickleback.

\section{Samples}

We used 50 randomly selected threespine sticklebacks from the same site near Bern, Switzerland. These fish were caught using hand nets in early December 2010. The dragonfly larvae (Aeshna spp.) for these experiments were caught using hand nets in early October 2010 from the same location and a pond nearby.

\section{Results}

Population based selection experiments

\section{Mortality}

The recapture rate (the ratio of the sum of the number of dead fish removed during the experiment and the number of surviving fish recovered at the end of the experiment to the total number of fish released at the start of the experiment) was $98.3 \%$ for the first run and $98.1 \%$ for the second run. In both runs, there was a much higher mortality in treatments (T1 and $\mathrm{T} 2$, respectively in runs 1 and 2) compared to controls (C1 and $\mathrm{C} 2$ ); mortality in T1 was $35.3 \%$ compared to $3.4 \%$ in $\mathrm{C} 1$, and $31.5 \%$ in T2 compared to $9.8 \%$ in $\mathrm{C} 2$ (both differences statistically significant; T1 vs. C1 $P=0.001$ and T2 vs. C2 $P=0.03$; MannWhitney $U$ tests).There was no significant difference in mortality between treatments from run 1 versus run 2 (T1 vs. T2; $P=0.460$ ), but significantly higher mortality occurred in C2 compared to $\mathrm{C} 1(P=0.009)$. Overall the mortality rate in our controls were comparable to those in a similar experiment conducted by Marchinko (2009).

\section{Linear morphological measurements}

All linear morphological measurements (SL, FSL, SSL, PSL, BD) as well as their size corrected residuals were normally distributed for both controls and treatment combined (Kolmogorov-Smirnov tests). Within treatments, only the residuals of FSL in the control ( $D=0.986, P=0.032)$ and the residuals of SSL in the predation treatment $(D=0.994$, $P=0.019$ ) were not normally distributed. The two first PC axes explain 52.4 and $24.0 \%$ 
Table 1 Statistical summary of the linear mixed models

\begin{tabular}{llr}
\hline Trait & $F_{1,737}$ & $P$ \\
\hline SL & 0.149 & 0.699 \\
FSL & 0.648 & 0.421 \\
SSL & 0.037 & 0.848 \\
PSL & 1.086 & 0.298 \\
BD & 5.370 & $\mathbf{0 . 0 2 1}$ \\
Plates & 0.031 & 0.860 \\
PC1 (explains 52.4 \% of total variance; trait loadings: & 0.192 \\
FSL, -0.495; SSL, -0.540; PSL, -0.629; BD, -0.259) & 1.706 & $\mathbf{0 . 0 4 4}$ \\
PC2 (explains 24.0\% of total variance; trait loadings: & & \\
FSL, -0.184; SSL, -0.214; PSL, -0.066; BD, 0.957) & 4.088 & \\
\hline
\end{tabular}

For each trait the control was tested against the treatment with ponds nested in runs as random factor to account for differences. Results for FSL, SSL, PSL and BD are based on size-corrected values. Statistically significant $P$ values are given in bold

of the total variation respectively. Loadings between these axes differed, as all spine lengths contributed most on the first and BD on the second axis.

None of the size corrected linear measurements except BD were significantly different between controls and treatments (Table 1$)$. BD showed a significant effect $\left(F_{1,737}=5.569\right.$, $P=0.019$ ) with surviving individuals in the treatment being deeper bodied than fish from the control. Concomitantly, the second PC axis, to which BD makes a very high contribution, showed a significant difference among treatments $\left(F_{1,737}=4.358, P=0.037\right)$. The first PC axis did not differ between control and treatment, nor did lateral plate counts or spine lengths. The distribution of morphological trait values for control and treatment fish for traits studied here, as well as the PC scores for linear traits along the first two PC axes are shown in Figs. 1 and S1.

\section{Lateral plate counts and coverage}

Lateral plate counts were strongly bimodal, and not normally distributed in either the treatments or the controls $(P<0.001$ in both cases; Kolmogorov-Smirnov tests). There was no significant overall difference in the number of lateral plates between controls and treatments $(P=0.798$; Mann-Whitney $U$ test) even when corrected for run and pond (Table 1). Plate cover, also not normally distributed, again showed no significant difference between treatments and controls $(P=0.810$; Mann-Whitney $U$ test). Plate cover was highly correlated with plate count $\left(R^{2}=0.845, P<0.001\right)$ in this population, which is not necessarily the case in other Swiss stickleback populations (O. Seehausen and K. Lucek, unpublished data).

\section{Stn382 allele frequencies}

Allele frequencies at the Stn382 locus were normally distributed among replicates $(P=0.699$, Kolmogorov-Smirnov test). The frequency of the $\mathrm{C}$-allele in the control was not significantly higher than in the treatments $(P=0.729$, one-tailed $t$ test; Fig. 2$)$. Further Stn382 C-alleles were not significantly higher in frequency in the surviving fish from treatments compared to the dead fish from the treatments, which were removed during the course of the experiment ( $P=0.400$, paired $t$ test; Fig. 2). 

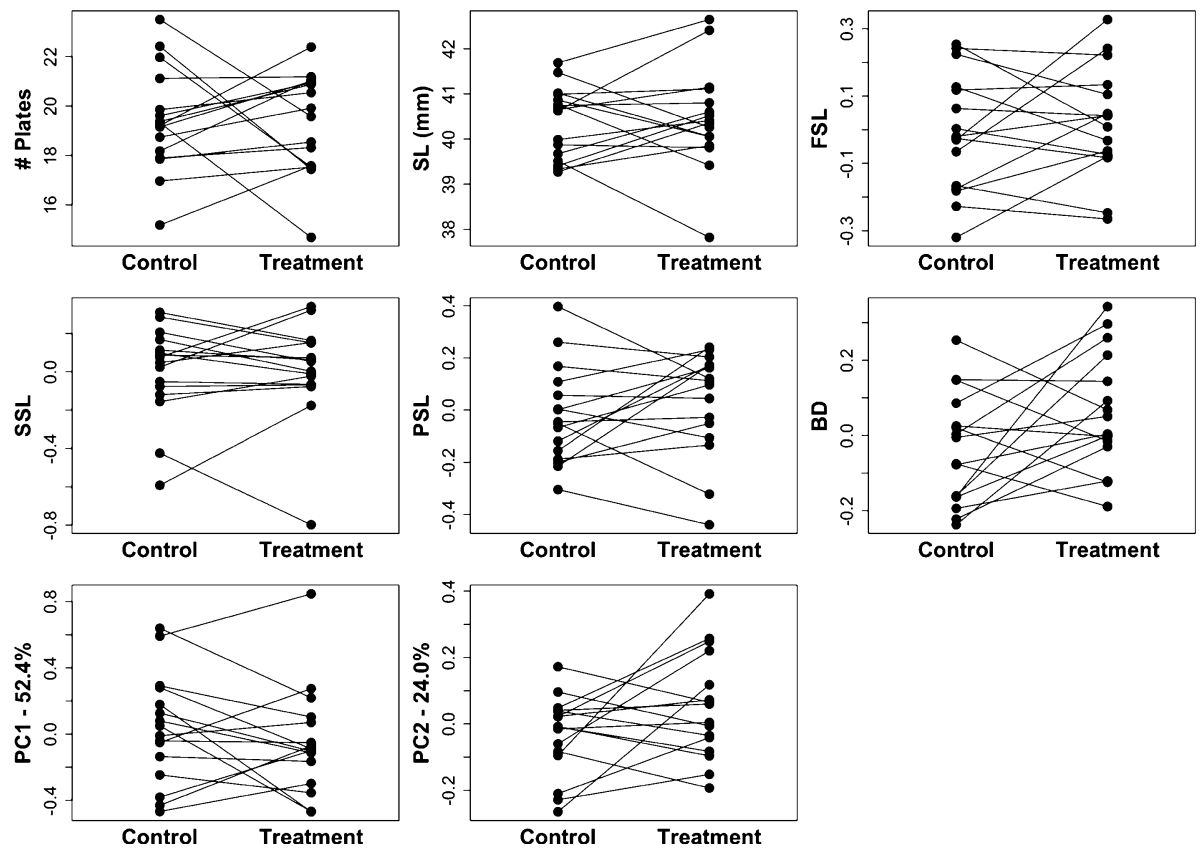

Fig. 1 Average trait values per replicate for both control and treatment for a the number of lateral plates, b SL (in mm); residuals of $\mathbf{c}$ first dorsal spine length, $\mathbf{d}$ second dorsal spine length, e pelvic spine length, f body depth on SL. In addition the average residuals per replicate of a principal component (PC) analysis using all size corrected linear morphological traits for the first $(\mathrm{g})$ and the second $(\mathrm{h})$ axis

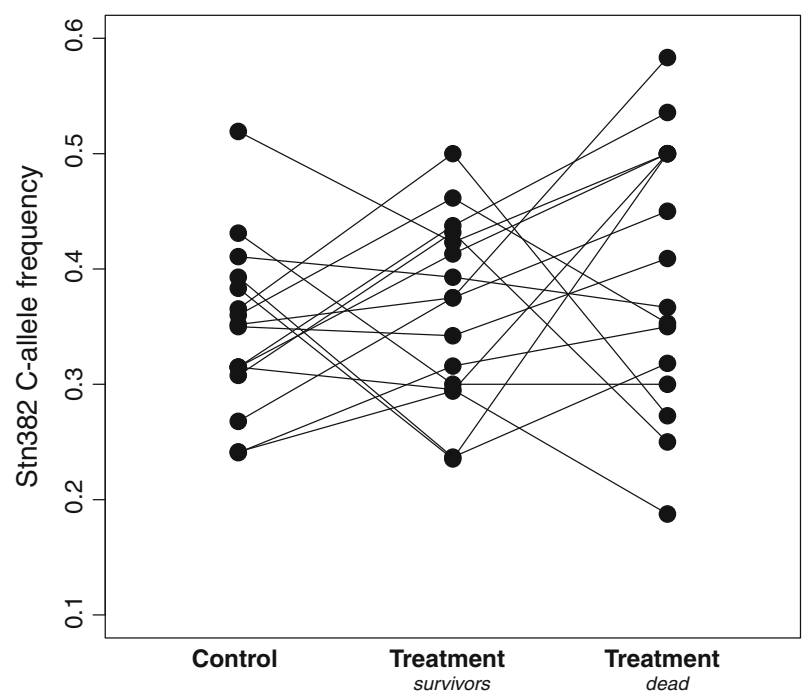

Fig. 2 Allele frequencies per replicate for the Stn382 C-allele in control and treatment fish that were alive at the end of the experiment ("survivors") and treatment fish that died during the experiment ("dead") 
Table 2 Sample sizes and $P$ values from comparisons (Fisher's exact test) of the Stn382 C-allele frequencies for each replicate

\begin{tabular}{lccrlc}
\hline Replicate & $N_{\text {Control }}$ & $N_{\text {Treatment }}$ & $N_{\text {Dead }}$ & $\begin{array}{l}\text { Control versus } \\
\text { treatment }\end{array}$ & $\begin{array}{l}\text { Treatment survivors } \\
\text { versus dead }\end{array}$ \\
\hline 1 & 27 & 19 & 10 & 0.956 & 0.505 \\
2 & 29 & 25 & 5 & $\mathbf{0 . 0 3 9}$ & 0.561 \\
3 & 30 & 17 & 12 & $\mathbf{0 . 0 2 3}$ & $\leq 0.001$ \\
4 & 30 & 19 & 11 & 0.500 & 0.191 \\
5 & 27 & 22 & 8 & 0.500 & $\mathbf{0 . 0 5 0}$ \\
6 & 28 & 19 & 11 & $\mathbf{0 . 0 1 6}$ & 0.135 \\
7 & 29 & 17 & 13 & 0.832 & 0.002 \\
8 & 27 & 16 & 14 & 0.980 & 0.101 \\
9 & 27 & 23 & 5 & 0.884 & 0.128 \\
10 & 26 & 26 & 4 & 0.101 & 0.160 \\
11 & 28 & 14 & 15 & 0.443 & 0.669 \\
12 & 28 & 20 & 10 & 0.965 & 0.195 \\
13 & 26 & 22 & 6 & 0.972 & $\mathbf{0 . 0 0 5}$ \\
14 & 24 & 13 & 17 & 0.943 & $\mathbf{0 . 0 2 9}$ \\
15 & 27 & 24 & 6 & 0.722 & 0.004 \\
16 & 26 & 19 & 11 & 0.043 & $\mathbf{0 . 0 5 0}$ \\
All & 439 & 315 & 158 & 0.500 & 0.331 \\
\hline
\end{tabular}

$N_{\text {Control }}$ number of fish in the control group, $N_{\text {Treatment }}$ number of fish in the treatment group that were alive at the end of the experiment, $N_{\text {Dead }}$ number of fish in the treatment group that died during the experiment For each replicate, two comparisons were made: between survivors of both control and treatment, and between the survivors from the treatment and the dead fish from the treatment. Values in bold indicate significantly different $\mathrm{C}$-allele frequencies in the predicted direction (i.e. control $>$ treatment and dead $>$ survivors) while italics indicate comparisons that were significant in the opposite direction. $P$ values $>0.05$ are denoted "ns"

Analysing the differences in Stn382 C-allele frequencies for each replicate separately, we found that the differences between replicates appeared to be stochastic. In only four out of 16 replicates did we find a significant difference in the allele frequency when comparing control and treatment for each replicate separately, using a Fisher exact test (Table 2). In three cases (replicates 2, 3 and 6) this difference was in the predicted direction, and in the fourth case (replicate 16) it was in the opposite direction. However, this test might be confounded by variation in starting frequencies so we conducted the same type of tests comparing the surviving fish from treatments with those which died during the experiment and found a significant difference in $\operatorname{Stn} 382$ allele frequencies in 7 of 16 replicates. In four cases (replicates 5, 13,14, and 16) the difference in allele frequencies was in the expected direction while in three cases (replicates $3,7,15$ ) it was in the opposite direction. Furthermore the difference in the Stn382 C-allele frequency of all replicates between "dead" and "alive" did not differ from zero $(P=0.400$, one sample $t$ test of difference against zero).

\section{Survival of Stn382 alleles and genotypes}

The survival for the two Stn382 alleles, as well as the three genotypes were measured in all the treatment replicates. The L-allele did not have a higher survival rate compared to the 

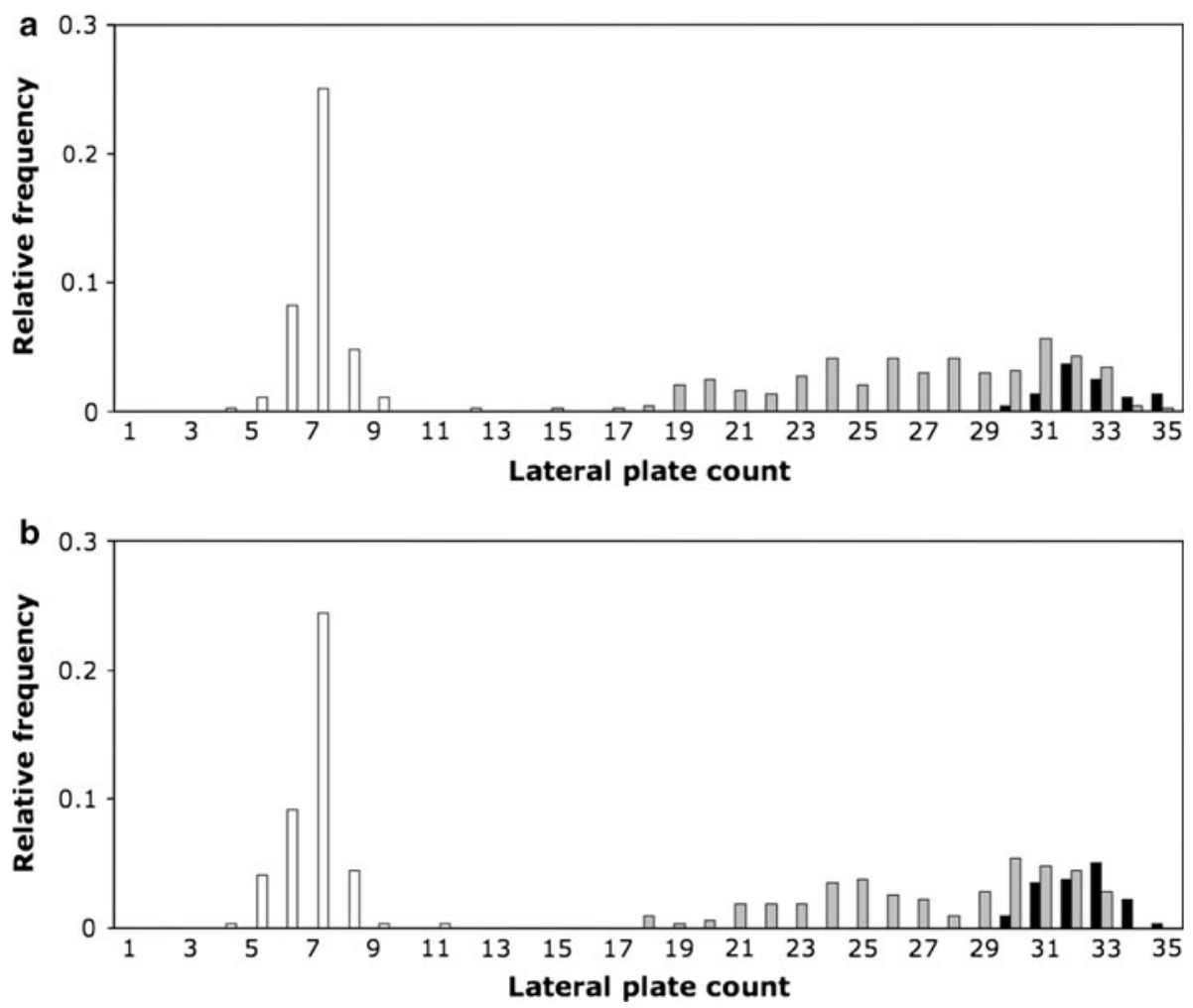

Fig. 3 Frequency distributions of lateral plate counts plotted for all three Stn382 genotypes in a controls and b treatments. Open bars: homozygote for the low plated (L-) allele, black bars: homozygote for the complete (C-) allele, grey bars: heterozygote

C-allele $(P=0.302$; one tailed $t$ test; Fig. S2). There was also no significant difference in the survival of the three genotypes (CC vs. LC: $P=0.728$; LC vs. LL: $P=0.275$; CC vs. LL: $P=0.737$; two-tailed $t$ tests; Fig. S2).

\section{Lateral plate count and Stn382 genotype}

There was no apparent difference in the frequency distribution of lateral plates, when broken down by $\operatorname{Stn} 382$ genotype, between the control and treatment fish (Fig. 3).

\section{Sex and age}

To test the possibility that the two sexes differ in the selective pressure exerted by predatory dragonfly larvae, we compared the sex ratios of fish in the treatments and controls, but found no significant difference $(P=0.960)$. We then asked whether there was a difference in age structure among fish in the controls and treatments, possibly reflecting learnt behavioural avoidance of predation. Again, we found no difference between controls and treatments (Fig. S3; $P=0.104$ ). 
Table 3 Selection differentials (i) for standard length (SL), first dorsal spine length (FSL), second dorsal spine length (SSL), pelvic spine length (PSL), body depth (BD), and lateral plate counts (plates) for each of the 16 replicates

\begin{tabular}{lrrrrrr}
\hline Replicate & \multicolumn{1}{c}{ SL } & \multicolumn{1}{c}{ FSL } & \multicolumn{1}{c}{ SSL } & \multicolumn{1}{c}{ PSL } & BD & Plates \\
\hline 1 & 0.11 & -0.11 & -1.01 & -0.37 & 0.55 & 0.22 \\
2 & 0.13 & $\mathbf{0 . 5 4}$ & $\mathbf{0 . 9 7}$ & 0.10 & 0.41 & -0.47 \\
3 & 0.28 & 0.19 & -0.04 & 0.34 & $\mathbf{0 . 6 8}$ & -0.39 \\
4 & 0.29 & -0.41 & -0.25 & -0.55 & -0.51 & 0.04 \\
5 & 0.42 & -0.32 & -0.34 & -0.15 & -0.01 & 0.06 \\
6 & 0.03 & -0.06 & -0.34 & 0.37 & $\mathbf{0 . 4 3}$ & -0.43 \\
7 & 0.02 & $\mathbf{0 . 9 2}$ & $\mathbf{0 . 6 5}$ & $\mathbf{0 . 6 2}$ & 0.11 & 0.05 \\
8 & -0.36 & 0.02 & -0.13 & 0.03 & 0.09 & 0.13 \\
9 & 0.14 & -0.21 & 0.28 & -0.21 & -0.24 & 0.10 \\
10 & -0.34 & -0.66 & -0.45 & -0.11 & -0.29 & -0.40 \\
11 & 0.29 & 0.50 & 0.47 & 0.42 & $\mathbf{0 . 5 7}$ & 0.12 \\
12 & 0.30 & -0.15 & -0.42 & -0.56 & -0.33 & 0.26 \\
13 & -0.29 & -0.05 & -0.03 & -0.02 & 0.46 & 0.01 \\
14 & -0.27 & -0.21 & 0.02 & 0.33 & $\mathbf{1 . 1 2}$ & 0.31 \\
15 & -0.02 & 0.29 & 0.28 & 0.22 & 0.22 & 0.06 \\
16 & -0.18 & $\mathbf{0 . 6 0}$ & 0.10 & $\mathbf{0 . 7 9}$ & -0.06 & 0.19 \\
\hline
\end{tabular}

Statistically significant values of $i(P<0.05 ; P$ values based on 1,000 resampling steps $)$ are in bold. Results for FSL, SSL, PSL and BD are based on size corrected values

\section{Selection differentials}

Estimated selection differentials were significantly different from zero only in a few cases among the six traits tested in sixteen replicates (FSL in three replicates, SSL in two replicates, PSL in two replicates and BD in four replicates; Table 3). This suggests that in this experiment there is no indication that dragonfly larvae caused directional selection pressure on any of these morphological traits.

\section{Individual based selection experiments}

Standard lengths (SL) of stickleback and length of dragonfly larvae as well as the difference between these lengths were normally distributed for all categories (KolmogorovSmirnov tests). In nearly half (23 out of 50) of the trials, there was no attempt by the predator to capture the fish (category 1). Of the remaining trials, where the predator attempted to capture the prey, in eight cases the attempt was unsuccessful (category 2), while in nineteen cases it was successful (category 3).

Fish did not differ significantly in size between successful and unsuccessful predation attempts (two-tailed $t$ tests; $P=0.394$ ), nor did it differ between cases where attempts were made or not (category 1 vs. $2, P=0.912$; 1 vs. $3, P=0.333$ ). The same was true for dragonfly larvae: successful predators were not significantly different in size from unsuccessful ones $(P=0.504)$ nor did size of the predator differ between cases where no attempt was made and ones where they were (category 1 vs. $2, P=0.502 ; 1$ vs. 3 , $P=0.959$ ). Finally, the size difference between the predator and prey (length of dragonfly larva minus SL of fish) also did not differ between categories 2 and $3(P=0.249)$, nor did 

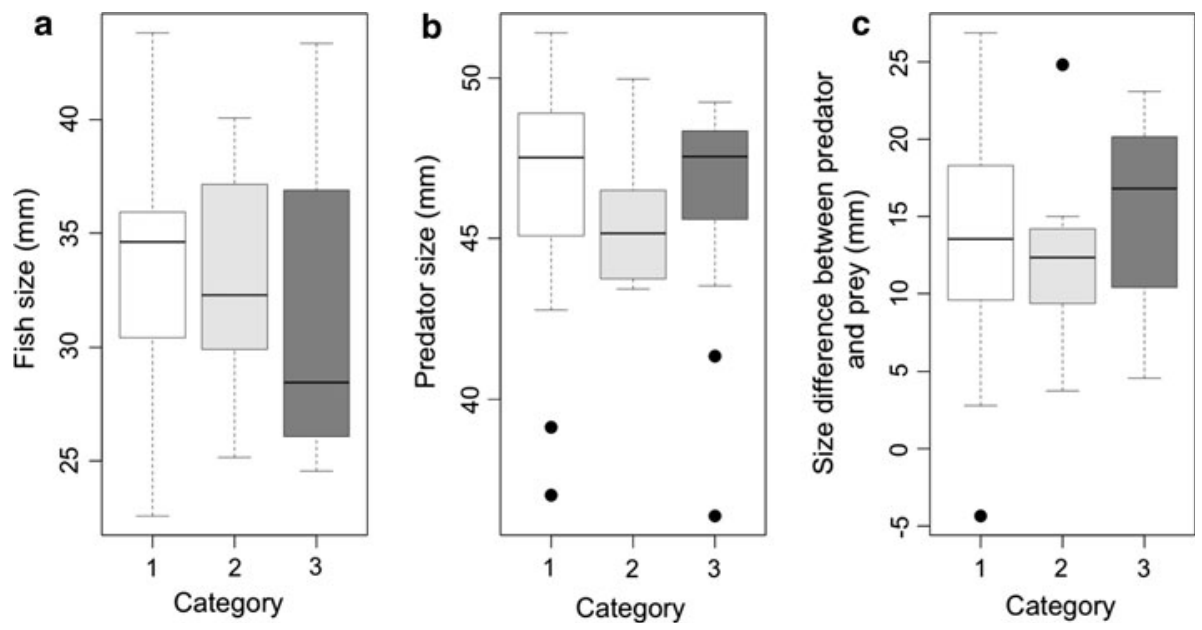

Fig. 4 Sizes of $\mathbf{a}$ fish and $\mathbf{b}$ dragonfly larvae, and $\mathbf{c}$ difference in size between predator and prey grouped by the outcome of individual-based experiments: 1 no attempted predation by the predator; 2 unsuccessful attempt by the predator and 3 successful attempt by the predator

it between category 1 and cases of attempted predation ( 1 vs. $2, P=0.697 ; 1$ vs. 3 , $P=0.412$; Fig. 4). Logistic regression using the outcome as a categorical response and difference in size between predator and prey as the predictor showed no significant relationship whether or not category 1 was included in the analysis $(P=0.637$ and 0.244 respectively).

\section{Discussion}

The aim of this study was to experimentally test predictions of the hypothesis that predation by dragonfly larvae exerts selection on stickleback armour traits by comparing the distribution of armour trait variants between different experimental conditions. We did not find any significant selective advantage for fish with reduced armour traits.

Reimchen (1980, 1994, 2000) hypothesized a reduction in armour from marine to freshwater habitats comprising three distinct components: a reduction in the posterior plates as an adaptation for increased burst velocity, a reduction in the anterior plates associated with a shift in predator behaviour from puncturing to compression and a reduction in spine lengths as an adaptation for increased post-capture escape against grappling predators. The hypothesis we addressed differed from the original hypothesis in that we tested whether dragonfly larvae exerted selection on the number of lateral plates and on the length of spines. We find no compelling evidence in this study that there is a selective advantage in the reduction of defense traits (spine lengths and lateral plate number).

We made 96 estimates of selection differentials for various armour traits: six traits, each measured in 16 replicates. In eleven of these cases, selection differentials differ from the null expectation. All of these cases point towards greater relative spine length and body depth in treatment survivors compared to control fish. Given that of 96 comparisons, about five may be expected to be significant by chance, but not in a predictable direction, the fact that eleven comparisons are significant in the same direction may be interpreted as 
evidence for selection for longer relative spine length and increased body depth. This trend for spine length is, however, contrary to the predictions of a selective advantage of reduced body armour under dragonfly predation. It is possible that fish with longer spines are attacked less often, and hence have enhanced survival, but our experiment does not allow us to unequivocally infer this. It might, however, be argued that a $P$ value of 0.05 is both arbitrary and too stringent to be applied to selection differentials, since only strong selection could be detected given our experimental sample sizes, whereas weak and moderate natural selection is most common in nature. Indeed the four highest selection differentials for plate number indicate a reduction in plate number in the treatment survivors relative to controls, consistent with predictions. This might be interpreted as weak evidence for a selective advantage of reduced plate cover. However, given our experimental design, sample sizes and use of standard statistical criteria, we cannot claim strong or unequivocal support for the hypothesis.

Marchinko (2009) showed that insect predation resulted in selection for faster growth rate, smaller spine size and selection for the allele associated with the low plate phenotype (Lallele), but could not demonstrate selection on the lateral plate phenotype itself. There are several differences between the two studies. Marchinko (2009) used a mix of dragonfly larvae (38\%) and Notonecta spp. (62\%) as predators for his experiments, while we used $100 \%$ dragonfly larvae. Marchinko (personal communication) also used smaller dragonfly larvae $(30-40 \mathrm{~mm})$ than the ones that we used $(36-51 \mathrm{~mm})$, which belong to the most common species in our study site. This difference in predator composition and size should be accounted for when comparing the two studies. Marchinko (2009) also found a higher survival probability for larger fish. In contrast, we found no selection on SL either in the population based experiments or in the individual based experiments, and did not observe a significant difference in the SL for surviving fish between control and treatment, although our results do suggest that larger body depth might provide a selective advantage to fish in the face of predation by dragonfly larvae. Our study does not rule out that predation by invertebrate predators other than large Aeshna spp. larvae could be a selective agent for faster growth or increased armour. That in our experiments the dragonfly larvae hunted randomly among all size classes of stickleback is in contrast with previous findings, where Aeshna larvae captured and consumed mostly juvenile stickleback up to a length of $25 \mathrm{~mm}$ (Foster et al. 1988). Taken together, our results give some indications that the reduction in armour traits as a defense against dragonfly larvae predation may not be a universal pattern in stickleback.

There is some evidence for a trade-off between growth rate and armour traits (Marchinko and Schluter 2007) as well as with the Eda gene, with the low allele conferring a growth advantage (Barrett et al. 2008). Since overwinter survival-a key component of fitness - is correlated with a high growth rate during summer (Curry et al. 2005), lateral plates are likely to lose their adaptive value in freshwater systems where gape-limited predators are less dominant. Therefore selection on growth could outweigh the role of being protected against gape-limited predators in freshwater. Consequently, armour might experience indirect selection resulting from direct selection on growth rate (Lahti et al. 2009). Further, Bergstrom (2002) found that an increased number of lateral plates is associated with reduced velocity and displacement during the fast-start escape response. This suggests that reduced plate cover could be advantageous in some predation regimes, such as those dominated by avian predators and ambush hunting fish. The match between phenotype and swimming performance also differs between lake and stream habitats, suggesting that this coupling has a genetic basis and might be involved in adaptive divergence (Hendry et al. 2010). 
Our experiments were conducted with fish from a population that is naturally highly polymorphic for armour related phenotypes. The fact that we used predators and prey from wild populations from the same location provides biological realism to this study. The fish from this population might have acquired behavioral or physiological traits, relevant to predator avoidance or escape as well as potentially coevolved predators, with adapted hunting strategies. Moreover, the polymorphic wild population allows us to rule out effects of negative epistatic effects on fitness, which may be common in crosses of divergent populations with different genetic background (Ungerer et al. 2003). The parallel instances of reduction in armour traits in the threespine stickleback during the colonization of freshwater systems are likely a result of the interaction of multiple selective forces, and not just of predation. We must note that the predator-prey relationship that we examined represents only a single link in a complex network of ecological interactions. Still, our data suggest that in the face of predation from large predatory insect larvae, a reduction in body armour may offer little advantage.

Acknowledgments The experiments reported in this study were approved by the veterinary office of the Canton of Bern and complied with all legal requirements in Switzerland (permit no. BE51/09). We thank the members of the Eawag Fish Ecology and Evolution lab for constructive feedback and two anonymous reviewers of previous versions of the manuscript for their comments.

\section{References}

Bańbura J (1994) Lateral plate morph differentiation of freshwater and marine populations of the threespined stickleback, Gasterosteus aculeatus, in Poland. J Fish Biol 44:773-783

Barrett RDH (2010) Adaptive evolution of lateral plates in three-spined stickleback Gasterosteus aculeatus: a case study in functional analysis of natural variation. J Fish Biol 77:311-328

Barrett R, Rogers S, Schluter D (2008) Natural selection on a major armor gene in threespine stickleback. Science 322:255-257

Bell MA, Foster SA (eds) (1994) The evolutionary biology of the threespine stickleback. Oxford University Press, New York

Bell MA, Orti G, Walker JA, Koenigs JP (1993) Evolution of pelvic reduction in threespine stickleback fish-a test of competing hypothesis. Evolution 47:906-914

Bergstrom CA (2002) Fast-start swimming performance and reduction in lateral plate number in threespine stickleback. Can J Zool 80:207-213

Berner D, Roesti M, Hendry AP, Salzburger W (2010) Constraints on speciation suggested by comparing lake-stream stickleback divergence across two continents. Mol Ecol 19:4963-4978

Bjærke O, Østbye K, Lampe HM, Vøllestad LA (2010) Covariation in shape and foraging behaviour in lateral plate morphs in the three-spined stickleback. Ecol Freshw Fish 19:249-256

Colosimo PF, Peichel CL, Nereng K, Blackman BK, Shapiro MD, Schluter D, Kingsley DM (2004) The genetic architecture of parallel armor plate reduction in threespine sticklebacks. PLoS Biol 2:e109

Colosimo PF, Hosemann KE, Balabhadra S, Villarreal G, Dickson M, Grimwood J, Schmutz J, Myers RM, Schluter D, Kingsley DM (2005) Widespread parallel evolution in sticklebacks by repeated fixation of Ectodysplasin alleles. Science 307:1928-1933

Conner J (2001) How strong is natural selection? Trends Ecol Evol 16:215-217

Cresko W, Amores A, Wilson C, Murphy J, Currey M, Phillips P, Bell M, Kimmel C, Postlethwait J (2004) Parallel genetic basis for repeated evolution of armor loss in Alaskan threespine stickleback populations. Proc Natl Acad Sci USA 101:6050-6055

Curry RA, Currie SL, Arndt SK, Bielak AT (2005) Winter survival of age-0 smallmouth bass, Micropterus dolomieu in north eastern lakes. Environ Biol Fishes 72:111-122

Endler JA (1986) Natural selection in the wild. Princeton University Press, Princeton

Ernande B, Dieckmann U, Heino M (2004) Adaptive changes in harvested populations: plasticity and evolution of age and size at maturation. Proc R Soc Lond B Biol Sci 271:415 
Foster SA, Garcia VB, Town MY (1988) Cannibalism as the cause of an ontogenetic shift in habitat use by fry of the threespine stickleback. Oecologia 74:577-585

Gårdmark A, Dieckmann U (2006) Disparate maturation adaptations to size-dependent mortality. Proc R Soc Lond B Biol Sci 273:2185

Giles N (1983) The possible role of environmental calcum levels during the evolution of phenotypic diversity in Outer Hebridean populations of the Three-spined stickleback, Gasterosteus aculeatus. J Zool (Lond) 199:535-544

Gross HP (1978) Natural selection by predators on the defensive apparatus of the three-spined stickleback, Gasterosteus aculeatus L. Can J Zool 56:398-413

Helfman GS, Collette BB, Facey DE (1997) The Diversity of Fishes. Blackwell Science, Inc, Hoboken, pp 141-145

Hendry AP, Hudson K, Walker JA, Räsänen K, Chapman LJ (2010) Genetic divergence in morphologyperformance mapping between Misty Lake and inlet stickleback. J Evol Biol 24:23-35

Heuts MJ (1947) The phenotypical variability of Gasterosteus aculeatus L. populations in Belgium. Mededel Koninkl Vlaam Akad Wetenschap 9:5-63

Hoogland R, Morris D, Tinbergen N (1956) The spines of sticklebacks (Gasterosteus and Pygosteus) as means of defence against predators (Perca and Esox). Behaviour 10:205-236

Johnson J (2001) Adaptive life-history evolution in the livebearing fish Brachyrhaphis rhabdophora: genetic basis for parallel divergence in age and size at maturity and a test of predator-induced plasticity. Evolution 55:1486-1491

Lahti DC, Johnson NA, Ajie BC, Otto SP, Hendry AP, Blumstein DT, Coss RG, Donohue K, Foster SA (2009) Relaxed selection in the wild. Trends Ecol Evol 24:487-496

Langerhans RB, Gifford ME, Joseph EO (2007) Ecological speciation in Gambusia fishes. Evolution 61:2056-2074

Lucek K, Roy D, Bezault E, Sivasundar A, Seehausen O (2010) Hybridization between distant lineages increases adaptive variation during a biological invasion: stickleback in Switzerland. Mol Ecol 19:3995-4011

Marchinko KB (2009) Predation's role in repeated phenotypic and genetic divergence of armor in threespine stickleback. Evolution 63:127-138

Marchinko K, Schluter D (2007) Parallel evolution by correlated response: lateral plate reduction in threespine stickleback. Evolution 61:1084-1090

McKinnon JS, Rundle HD (2002) Speciation in nature: the threespine stickleback model systems. Trends Ecol Evol 17:480-488

Munzing J (1959) Biologie, Variabilitat, und genetik von Gasterosteus aculeatus L. (Pisces). Untersuchungen im Elbegebiet. Int Revue Gesamten Hydrobiol 44:317-382

Myhre F, Klepaker T (2009) Body armour and lateral-plate reduction in freshwater three-spined stickleback Gasterosteus aculeatus: adaptations to a different buoyancy regime? J Fish Biol 75:2062-2074

Peichel CL, Nereng KS, Ohgi KA, Cole BL, Colosimo PF, Buerkle CA, Schluter D, Kingsley DM (2001) The genetic architecture of divergence between threespine stickleback species. Nature 414:901-905

Peichel CL, Ross JA, Matson CK, Dickson M, Grimwood J, Schmutz J, Myers RM, Mori S, Schluter D, Kingsley DM (2004) The master sex-determination locus in threespine sticklebacks is on a nascent Y chromosome. Curr Biol 14:1416-1424

Reimchen TE (1980) Spine deficiency and polymorphism in a population of Gasterosteus aculeatus-an adaptation to predators? Can J Zool 58:1232-1244

Reimchen TE (1992a) Extended longevity in a large-bodied stickleback, Gasterosteus, population. Can Field-Nat 106:122-125

Reimchen TE (1992b) Injuries on stickleback from attacks by a toothed predator (Oncorhynchus) and implications for the evolution of lateral plates. Evolution 46:1224-1230

Reimchen TE (1994) Predators and morphological evolution in threespine stickleback.The Evolutionary Biology of the Threespine Stickleback. M. A. Bell and S. A. Foster, Oxford University Press, New York, pp 240-276

Reimchen TE (2000) Predator handling failures of lateral plate morphs in Gasterosteus aculeatus: functional implications for the ancestral plate condition. Behaviour 137:1081-1096

Reznick D, Endler J (1982) The impact of predation on life history evolution in Trinidadian guppies (Poecilia reticulata). Evolution 36:160-177

Rundle HD, Nosil P (2005) Ecological speciation. Ecol Lett 8:336-352

Schluter D (2000) The ecology of adaptive radiation. Oxford University Press, New York

Schluter D, Conte GL (2009) Genetics and ecological speciation. Proc Natl Acad Sci USA 106:9955

Ungerer MC, Linder CR, Rieseberg LH (2003) Effects of genetic background on response to selection in experimental populations of Arabidopsis thaliana. Genetics 163:277-286 
Vamosi S (2002) Predation sharpens the adaptive peaks: survival trade-offs in sympatric sticklebacks. Ann Zool Fenn 39:237-248

Vamosi S (2005) On the role of enemies in divergence and diversification of prey: a review and synthesis. Can J Zool 83:894-910

Walsh M, Reznick D (2009) Phenotypic diversification across an environmental gradient: a role for predators and resource availability on the evolution of life histories. Evolution 63:3201-3213 